\title{
Short communication: Evaluation of MALDI-TOF mass spectrometry and a custom reference spectra expanded database for the identification of bovine-associated coagulase-negative staphylococci
}

\author{
M. Cameron, ${ }^{\star 1}$ J. Perry,† J. R. Middleton, $\ddagger$ M. Chaffer, ${ }^{\star}$ J. Lewis, $§$ and G. P. Keefe* \\ *Department of Health Management, Atlantic Veterinary College, University of Prince Edward Island, Charlottetown, Prince Edward Island, \\ Canada C1A 4P3 \\ †White Valley Veterinary Services, Lumby, British Columbia, Canada V0E 2G0 \\ ‡Department of Veterinary Medicine and Surgery, University of Missouri, Columbia 65211 \\ §Department of Pathology and Microbiology, Atlantic Veterinary College, University of Prince Edward Island, Charlottetown, Prince Edward Island, \\ Canada C1A 4P3
}

\section{ABSTRACT}

This study evaluated MALDI-TOF mass spectrometry and a custom reference spectra expanded database for the identification of bovine-associated coagulasenegative staphylococci (CNS). A total of 861 CNS isolates were used in the study, covering 21 different CNS species. The majority of the isolates were previously identified by rpoB gene sequencing $(\mathrm{n}=804)$ and the remainder were identified by sequencing of $h s p 60$ $(\mathrm{n}=56)$ and $t u f(\mathrm{n}=1)$. The genotypic identification was considered the gold standard identification. Using a direct transfer protocol and the existing commercial database, MALDI-TOF mass spectrometry showed a typeability of $96.5 \%(831 / 861)$ and an accuracy of $99.2 \%$ (824/831). Using a custom reference spectra expanded database, which included an additional 13 in-house created reference spectra, isolates were identified by MALDI-TOF mass spectrometry with $99.2 \%$ (854/861) typeability and $99.4 \%$ (849/854) accuracy. Overall, MALDI-TOF mass spectrometry using the direct transfer method was shown to be a highly reliable tool for the identification of bovine-associated CNS.

Key words: bovine coagulase-negative staphylococci, matrix-assisted laser desorption/ionization time of flight mass spectrometry, species identification

\section{Short Communication}

Matrix-assisted laser desorption/ionization time-offlight MS is a rapid and robust, cost-effective technology that can be used for bacterial identification and is being adopted by an increasing number of diagnostic laboratories (Kliem and Sauer, 2012). By means of

Received May 24, 2017.

Accepted August 30, 2017.

${ }^{1}$ Corresponding author: mcameron@upei.ca
MALDI-TOF MS, bacteria are identified via their unique protein profiles (mass spectrum) which are generated by the instrument and then matched to a reference database of known microbial spectra (termed Main SPectra; MSP). Coagulase-negative staphylococci are a group of bacteria frequently associated with bovine IMI. Despite the fact that the CNS species have vast differences in pathogenicity, ecology, and epidemiology (Sampimon et al., 2011; Supré et al., 2011; Vanderhaeghen et al., 2014), they are rarely classified to the species level due to issues of accuracy and high cost (Zadoks and Watts, 2009; Ajitkumar et al., 2013). In a recent study, we evaluated MALDI-TOF MS for the identification of bovine-associated CNS (Cameron et al., 2017). As part of that study, we identified gaps with respect to CNS species classification in the commercial microbial spectral database and filled those gaps with 13 in-house custom MSP, covering 8 species of CNS. When we tested the in-house expanded database against a collection of $226 \mathrm{CNS}$ isolates covering 16 species, MALDI-TOF MS displayed a typeability and accuracy of $100 \%$. The goal of the current study was to validate MALDI-TOF MS equipped with custom MSP in addition to the commercial database with a large collection of CNS field isolates.

For this study, 861 isolates covering 21 different species of CNS belonging to the Mastitis Pathogen Culture Collection of the Canadian Bovine Mastitis and Milk Quality Research Network (Faculté de Médecine Vétérinaire, Université de Montréal, Saint-Hyacinthe, Quebec, Canada) were used (Table 1). These isolates had been previously identified by rpo $B$ gene sequencing with $\geq 94 \%$ sequence homology to the GenBank database with $\geq 5 \%$ difference from the next identified species (Fry et al., 2014). A subset of these isolates was also submitted for hsp60 gene sequencing and one isolate was submitted for tuf sequencing (Supplemental Table S1; https://doi.org/10.3168/jds.2017-13226). Stock iso- 
lates, which were stored at $-80^{\circ} \mathrm{C}$, were thawed at room temperature for $15 \mathrm{~min}$. Columbia agar plates containing $5 \%$ sheep blood were streaked with a $1-\mu \mathrm{L}$ sterile disposable loop to obtain isolated colonies. Plates were incubated for 18 to $24 \mathrm{~h}$ at $35^{\circ} \mathrm{C}$. The following day, cultures were examined for growth and purity. Single colonies were sub-cultured onto blood agar plates and incubated for 18 to $24 \mathrm{~h}$ at $35^{\circ} \mathrm{C}$.

Isolate classifications were carried out using the direct transfer method as previously described (Cameron et al., 2017). Briefly, a single-use, 15-cm sterile wooden applicator stick was used to lift material from a well-isolated bacterial colony followed by smearing a thin film of colony material onto a ground steel MSP 96-spot target (Bruker Daltonics Inc., Billerica, MA) in duplicate. The spots were allowed to air dry at room temperature. Subsequently, the spots were overlaid with $1.0 \mu \mathrm{L}$ of a saturated solution of $\alpha$-cyano-4-hydroxycinnamic acid matrix in $50 \%$ acetonitrile, $47.5 \%$ water, and $2.5 \%$ trifluoroacetic acid (Sigma-Aldrich Canada Inc., Oakville, ON, Canada) using single-use pipette tips and air dried at room temperature. All targets were calibrated using the Bacterial Test Standard (Bruker Daltonics Inc.) and included Staphylococcus aureus ssp. aureus ATCC 29213 and Escherichia coli ATCC 25922 control classification samples in duplicate. To confirm that target

Table 1. Collection of 21 species of CNS analyzed by MALDI-TOF MS

\begin{tabular}{|c|c|c|}
\hline Species $^{1}$ & $\mathrm{n}$ & $\%$ of total \\
\hline Staphylococcus arlettae & 4 & $<1$ \\
\hline Staphylococcus aureus & 1 & $<1$ \\
\hline Staphylococcus auricularis & 1 & $<1$ \\
\hline Staphylococcus capitis & 7 & 1 \\
\hline Staphylococcus chromogenes & 401 & 47 \\
\hline Staphylococcus cohnii & 24 & 3 \\
\hline Staphylococcus epidermidis & 34 & 4 \\
\hline Staphylococcus equorum & 3 & $<1$ \\
\hline Staphylococcus gallinarum & 4 & $<1$ \\
\hline Staphylococcus haemolyticus & 66 & 8 \\
\hline Staphylococcus hominis & 1 & $<1$ \\
\hline Staphylococcus hyicus & 9 & 1 \\
\hline Staphylococcus nepalensis & 2 & $<1$ \\
\hline Staphylococcus pasteuri & 3 & $<1$ \\
\hline Staphylococcus saprophyticus & 7 & 1 \\
\hline Staphylococcus sciuri & 10 & 1 \\
\hline Staphylococcus simulans $^{2}$ & 170 & 20 \\
\hline Staphylococcus succinus & 4 & $<1$ \\
\hline Staphylococcus vitulinus & 1 & $<1$ \\
\hline Staphylococcus warneri & 9 & 1 \\
\hline Staphylococcus xylosus & 100 & 12 \\
\hline Total & 861 & 100 \\
\hline
\end{tabular}

${ }^{1}$ Determined by rpoB (804/861), hsp60 (56/861), or tuf (1/861) gene sequencing

${ }^{2}$ One isolate of $S$. simulans resolved to the Staphylococcus carnosus group level only (includes $S$. carnosus, S. condimenti, S. massiliensis, S. piscifermentans, and S. simulans). cleaning was effective and no residual bacterial material from a previous run remained, one spot on each target contained only matrix with no bacterial sample.

All bacterial spectral captures and classifications were carried out using Bruker Daltonics Research Use Only microbial classification platform that included a Microflex LT MS, flexControl software (version 3.4), MALDI Biotyper Real Time Classification (RTC) and Offline Classification (OC; version 3.1) with a 5,627 MSP reference database library (MBT-BDAL-5627). The MALDI Biotyper RTC was carried out according to MALDI Biotyper 3.1 User Manual and parameter settings as previously published (Cameron et al., 2017). Isolates were initially classified in real time against the existing commercial database. Archived spectra from RTC jobs were re-classified using Biotyper OC identification standard method (version 1.1 with default parameter settings) against the commercial database with the addition of 11 CNS custom MSP previously described (Cameron et al., 2017) and an additional 2 custom MSP, all described in Supplemental Table S2 (https://doi.org/10.3168/jds.2017-13226). The manufacturer's recommendations for identification score interpretation are as follows: a score of 2.000 to 3.000 is considered probable to highly probable species identification, a score of 1.700 to 1.999 indicates probable genus identification, and a score of $<1.700$ is considered not reliable identification. Discordant identifications were defined as identifications by MALDI-TOF MS that did not match the gene sequencing-based identification. Isolates were initially run in duplicate. Isolates failing to achieve a score of $\geq 1.700$ (not reliable identification) on either of the 2 replicates were re-classified in a second round of duplicate direct transfer classifications of new 18 to $24 \mathrm{~h}$ cultures originating from the stock isolates using the same procedures described above. Isolates that remained unidentified were analyzed using a tube formic acid extraction procedure as previously described (Cameron et al., 2017). Finally, isolates with discordant identifications were resolved by hsp60 gene sequencing. To ensure that the sample analyzed by MALDI-TOF MS was the same as the sample submitted for sequencing, for each sample, a single isolated colony was spotted onto the target in duplicate and a portion of that same colony was streaked onto a new blood agar plate to create bacterial colonies for DNA extraction and sequencing.

The highest MALDI-TOF Biotyper classification score among the replicates of an isolate was considered the final identification and score. The performance of MALDI-TOF MS was evaluated in terms of its typeability and accuracy. In our previous work, lowering the species-level identification threshold to 1.700 improved 
species-level identification rates with only minimal effect on the accuracy (Cameron et al., 2017). Thus, in the current study, typeability refers to the ability of MALDI-TOF MS to assign a species name to a sample with an identification score $\geq 1.700$ and accuracy is the ability of MALDI-TOF MS to obtain the correct species name for a sample, considering the gene sequencing identification to be the gold standard identity of an isolate.

After the first round of duplicate direct transferbased analysis, 40 isolates were not identified and were subjected to a second round of duplicate direct transfer classifications which resulted in the identification of all but 7 isolates. A total of 57 isolates with discordant identifications were submitted for $h s p 60$ gene sequencing. Of that collection, one isolate failed to be resolved by hsp60 gene sequencing (closest match Staphylococcus simulans with sequence homology of $89 \%$ ). When evaluated further with tuf gene sequencing (Heikens et al., 2005), the closest match was Staphylococcus carnosus ssp. carnosus (sequence homology 97.4\%) and the next closest species match Staphylococcus condimenti (sequence homology 97.2\%). The reference identity for this isolate was considered only to the group level [i.e., S. carnosus group (includes S. carnosus, S. condimenti, Staphylococcus massiliensis, Staphylococcus piscifermentans, and Staphylococcus simulans; Tang et al., 2014)]. We found $100 \%$ agreement between the MALDI-TOF MS classification and the hsp60/tuf identification for this collection of 57 isolates (Supplemental Table S2; https://doi.org/10.3168/jds.2017-13226). Our previous work showed that for isolates with initially discordant results between the reference and MALDI-TOF MS, the results of sequencing of rpoB and hsp60 were in agreement, and concordant with the MALDI-TOF MS-based identification (Cameron et al., 2017). As discussed in the earlier paper, initial discordant results between reference and MALDI-TOF MS that were later resolved in favor of MALDI-TOF MS with further genotypic examination may be the result of errors in labeling or mixed growth in stock isolates (Cameron et al., 2017). Thus, for this collection of 57 isolates, the identifications based upon the updated hsp60 or tuf sequencing were used as the reference identification for the evaluation of MALDI-TOF MS.

Using the 1.700 cut-off and considering only the direct transfer-based classifications, when matched against the existing commercial database, isolates were identified by MALDI-TOF MS with a typeability of $96.5 \%(831 / 861)$ and an accuracy of $99.2 \%$ (824/831), whereas only $69.9 \%(602 / 861)$ of the isolates achieved reliable species level score using the manufacturer's threshold (i.e., $\geq 2.000$ ). When isolates were classified against the custom MSP expanded database, MALDITOF MS achieved a typeability of $99.2 \%(854 / 861)$ and an accuracy of $99.4 \%(849 / 854)$ at the 1.700 threshold, whereas only $77.6 \%(668 / 861)$ of the isolates achieved reliable species level score using the manufacturer's threshold (i.e., $\geq 2.000$ ). The final results for the custom MSP expanded database by CNS species using the direct transfer protocol are presented in Table 2. Five isolates of Staphylococcus hyicus were misidentified as Staphylococcus chromogenes (ID score range 1.700 to $<2.000$ ). For 4 of the 5 isolates of $S$. hyicus that were misidentified, the second closest match was in fact $S$. hyicus and within the same ID score level (i.e., 1.700 to $<2.000$ ). When MALDI-TOF ranked identifications show the same ID score level for $>1$ species, such as the case for these 4 isolates of $S$. hyicus, results should be considered classifications with poor discrimination. These 5 isolates of $S$. hyicus with discordant/poor discriminatory results and the 7 isolates that remained unidentified after a second round of duplicate direct transfer classifications were further examined using formic acid extraction procedures with results presented in Table 3.

According to our previous study and further supported by the results presented here, MALDI-TOF MS using a direct transfer protocol is a reliable tool for the identification of bovine-associated CNS (Cameron et al., 2017). The addition of user-created custom MSP to improve CNS species coverage of the reference spectral database improved the performance of the instrument in terms of typeability (from 96.5 to $99.2 \%$ ). The commercial reference database did not have an entry for S. gallinarum, and thus the instrument was unable to identify these isolates when relying on the commercial database. Others have shown that species redundancy in the database is positively associated with sample identification, as multiple reference spectra are often required to capture the diversity that exists among the isolates of a single bacterial species (Lartigue et al., 2009; Seng et al., 2009; Alatoom et al., 2011).

Commercial database MSP are invariably created from spectra collected from formic acid protein extractions of colonies to obtain high-quality library entries. In the diagnostic laboratory setting, protein extraction of bacterial colonies, which is a multi-step process, can be considered impractical and thus a direct transfer of colonies approach is often preferred (McElvania Tekippe et al., 2013). Protein extracted and direct transfer spectral repertoire variations do occur with the same isolate. The extent of this direct transfer versus colony extracted spectral divergence varies depending on species. A consequence of this spectral divergence is a general lowering of classification scores when using 
Table 2. Direct transfer-based identification of 861 CNS isolates by MALDI-TOF MS and the in-house expanded Biotyper database (Bruker Daltonics Inc., Billerica, MA) compared with rpoB (804/861), hsp60 (56/861), and tuf (1/861) gene sequencing

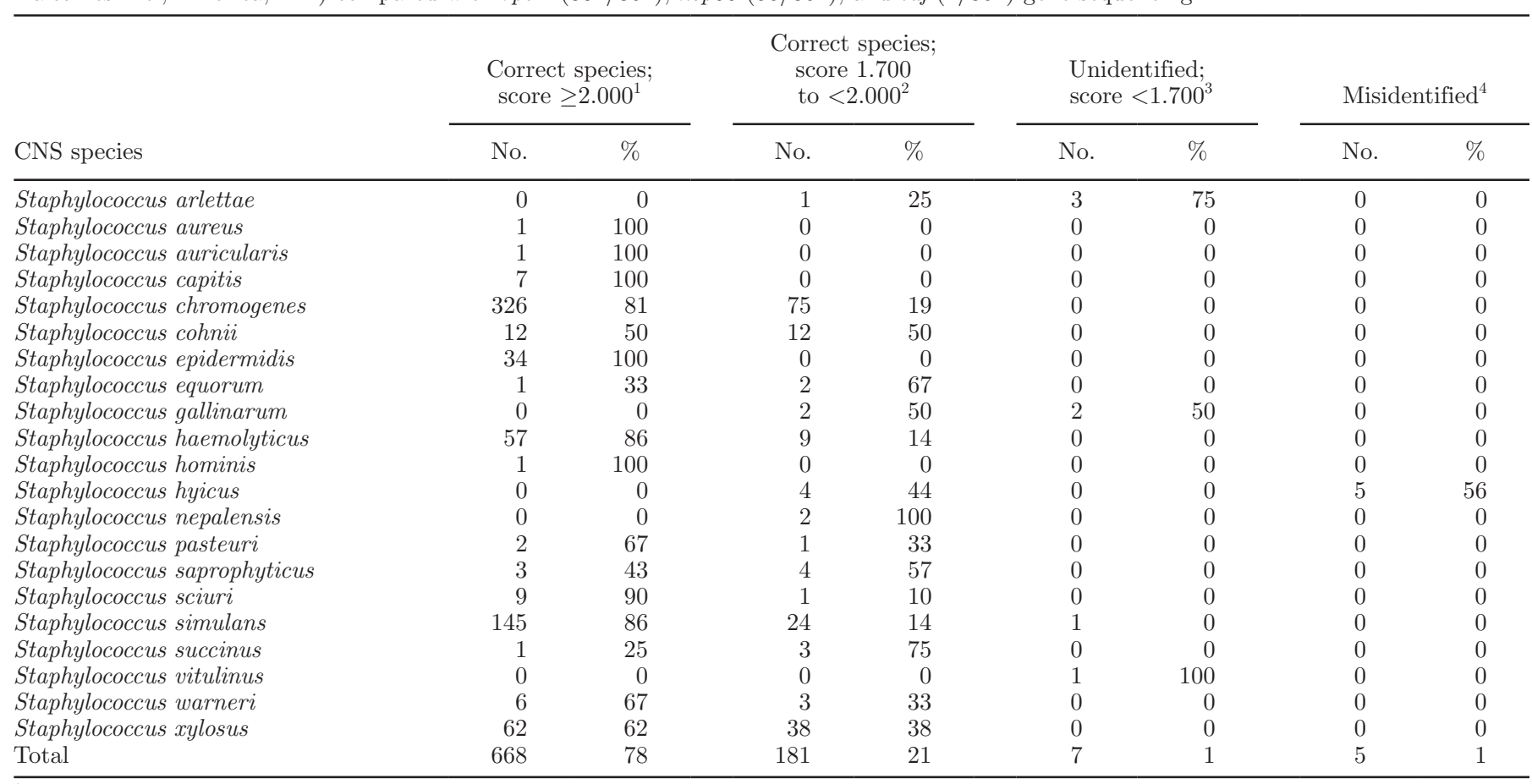

${ }^{1}$ Manufacturer's score interpretation: reliable identification to the species level.

${ }^{2}$ Manufacturer's score interpretation: reliable identification to the genus level.

${ }^{3}$ Manufacturer's score interpretation: no reliable identification.

${ }^{4}$ MALDI-TOF MS species is not the same as the reference.

the direct colony transfer method. In many cases, the spectral divergence is minimal and the score reduction is inconsequential. However, for certain species, the divergence is very significant and scores can be consistently reduced to $<1.700$. According to the results of the current study, accepting a lower score cutpoint (e.g., 1.700) for reliable species level identification would not affect the accuracy of bovine-associated CNS species identification. At worst, a direct transfer-based sample's spectral peak intensity is suppressed to the extent that identification by MALDI-TOF MS is not possible. This was the case for 7 isolates in the current study that remained unidentified by the direct transfer protocol, but were correctly classified using protein extraction processing. A potential solution would be to create direct transfer-based MSP to be used when a direct transfer protocol is used, but this approach would require full validation.

With regard to the discordant and poor discriminatory results for S. hyicus, S. hyicus and S. chromogenes were once subspecies of the species $S$. hyicus (Devriese et al., 1978), and formic acid extraction processing was able to resolve this issue. As noted in our previous paper, discordant and poor discriminatory results can

Table 3. Results of direct transfer and formic acid protein extraction sample processing for the identification of 12 CNS isolates by MALDI-TOF MS and the in-house expanded Biotyper database (Bruker Daltonics Inc., Billerica, MA)

\begin{tabular}{|c|c|c|c|c|c|}
\hline \multirow[b]{2}{*}{$\begin{array}{l}\text { No. of } \\
\text { isolates }\end{array}$} & \multirow[b]{2}{*}{ CNS species } & \multicolumn{4}{|c|}{ MALDI-TOF MS identification } \\
\hline & & Direct transfer ID & $\begin{array}{l}\text { Direct transfer } \\
\text { score }\end{array}$ & $\begin{array}{l}\text { Protein } \\
\text { extraction ID }\end{array}$ & $\begin{array}{c}\text { Protein extraction } \\
\text { score }\end{array}$ \\
\hline 3 & Staphylococcus arlettae & Not reliable identification & $<1.700$ & S. arlettae & $>2.000$ \\
\hline 2 & Staphylococcus gallinarum & Not reliable identification & $<1.700$ & S. gallinarum & $>2.000$ \\
\hline 5 & Staphylococcus hyicus & Staphylococcus chromogenes & 1.700 to $<2.000$ & S. hyicus & $>2.000$ \\
\hline 1 & Staphylococcus simulans & Not reliable identification & $<1.700$ & S. simulans & 1.700 to $<2.000$ \\
\hline 1 & Staphylococcus vitulinus & Not reliable identification & $<1.700$ & S. vitulinus & $>2.000$ \\
\hline
\end{tabular}


occur when related bacterial species have very similar spectral profiles, making it difficult for MALDI-TOF MS to classify correctly (Cameron et al., 2017). In our experience, creation of high-quality MSP entries for these problem species or strains can minimize or eliminate classification problems.

Staphylococcus agnetis is a newly described species and is genetically similar to S. hyicus (Taponen et al., 2012). The commercial reference database used in this study did not have an entry for S. agnetis. Staphylococcus agnetis had not been fully described at the time of the original rpoB sequencing of the collection of CNS considered in the current study. Subsequent to the MALDI-TOF MS classification of this CNS collection, whole-genome sequencing information became available for many isolates of the collection, including all 9 isolates of $S$. hyicus. Whole-genome sequencing revealed that 6 of the $9 S$. hyicus were more correctly identified as $S$. agnetis (J. de Buck, Department of Production Animal Health, Faculty of Veterinary Medicine, University of Calgary, Calgary, Alberta, Canada, personal communication). Follow-up spectral review of $S$. agnetis and $S$. hyicus identified 4 to 6 putative $S$. agnetis-unique marker peaks and suggests the potential for differentiation of $S$. agnetis and S. hyicus by MALDI-TOF MS (J. Lewis, personal communication). Development and validation of an S. agnetis custom MSP is a priority for future work.

The results presented in this paper, combined with those presented previously, show that MALDI-TOF MS is an accurate tool for the identification of bovineassociated CNS. Advantages of MALDI-TOF MS include the ability to create custom MSP to enhance the performance of the instrument and reduction of the reliable species-level identification score interpretation criteria without negative effect on accuracy. Current limitations of the tool when used with a direct transfer protocol include poor discrimination between isolates of S. hyicus with isolates of S. chromogenes (and likely $S$. agnetis), and the potential lack of identification for certain bovine-associated CNS species (notably Staphylococcus arlettae, Staphylococcus gallinarum, and Staphylococcus vitulinus).

\section{ACKNOWLEDGMENTS}

The authors thank Béatrice Després, Atlantic Veterinary College MALDI-TOF mass spectrometry Classification Research and Development Unit (Charlottetown, Prince Edward Island, Canada), and Natasha Robinson, Maritime Quality Milk (University of Prince Edward Island, Charlottetown, Prince Edward Island, Canada), for their technical assistance. Thanks go to
Pamela Adkins (University of Missouri, Columbia) for her assistance in reviewing the manuscript. This research is supported in main part by Agriculture and Agri-Food Canada (Ottawa, ON), and by additional contributions from Dairy Farmers of Canada (Ottawa, ON), the Canadian Dairy Network (Guelph, ON), and the Canadian Dairy Commission (Ottawa, ON) under the Agri-Science Clusters Initiative. As per the research agreement, aside from providing financial support, the funders have no role in the design and conduct of the studies, data collection and analysis, or interpretation of the data. Researchers maintain independence in conducting their studies, own their data, and report the outcomes regardless of the results. The decision to publish the findings rests solely with the researchers.

\section{REFERENCES}

Ajitkumar, P., H. W. Barkema, R. N. Zadoks, D. W. Morck, F. J. U. M. van der Meer, and J. De Buck. 2013. High-resolution melt analysis for species identification of coagulase-negative staphylococci derived from bovine milk. Diagn. Microbiol. Infect. Dis. 75:227-234. https://doi.org/10.1016/j.diagmicrobio.2012.11.008.

Alatoom, A. A., S. A. Cunningham, S. M. Ihde, J. Mandrekar, and R. Patel. 2011. Comparison of direct colony method versus extraction method for identification of Gram-positive cocci by use of Bruker Biotyper Matrix-Assisted Laser Desorption Ionization-Time of Flight mass spectrometry. J. Clin. Microbiol. 49:2868-2873. https://doi.org/10.1128/JCM.00506-11.

Cameron, M., H. W. Barkema, J. De Buck, S. De Vliegher, M. Chaffer, J. Lewis, and G. P. Keefe. 2017. Identification of bovine-associated coagulase-negative staphylococci by matrix-assisted laser desorption/ionization time-of-flight mass spectrometry using a direct transfer protocol. J. Dairy Sci. 100:2137-2147. https://doi.org/10 $.3168 /$ jds.2016-12020.

Devriese, L. A., V. Hajek, P. Oeding, S. A. Meyer, and K. H. Schleifer. 1978. Staphylococcus hyicus (Sompolinsky 1953) comb. nov. and Staphylococcus hyicus ssp. chromogenes ssp. nov. Int. J. Syst. Evol. Microbiol. 28:482-490.

Fry, P. R., J. R. Middleton, S. Dufour, J. Perry, D. Scholl, and I. Dohoo. 2014. Association of coagulase-negative staphylococcal species, mammary quarter milk somatic cell count, and persistence of intramammary infection in dairy cattle. J. Dairy Sci. 97:48764885. https://doi.org/10.3168/jds.2013-7657.

Heikens, E., A. Fleer, A. Paauw, A. Florijn, and A. C. Fluit. 2005. Comparison of genotypic and phenotypic methods for species-level identification of clinical isolates of coagulase-negative staphylococci. J. Clin. Microbiol. 43:2286-2290. https://doi.org/10.1128/ JCM.43.5.2286-2290.2005.

Kliem, M., and S. Sauer. 2012. The essence on mass spectrometry based microbial diagnostics. Curr. Opin. Microbiol. 15:397-402. https://doi.org/10.1016/j.mib.2012.02.006.

Lartigue, M.-F., G. Hery-Arnaud, E. Haguenoer, A.-S. Domelier, P.O. Schmit, N. van der Mee-Marquet, P. Lanotte, L. Mereghetti, M. Kostrzewa, and R. Quentin. 2009. Identification of Streptococcus agalactiae isolates from various phylogenetic lineages by Matrix-Assisted Laser Desorption Ionization-Time of Flight Mass Spectrometry. J. Clin. Microbiol. 47:2284-2287. https://doi.org/10 .1128/JCM.00175-09.

McElvania Tekippe, E., S. Shuey, D. W. Winkler, M. A. Butler, and C.-A. D. Burnham. 2013. Optimizing identification of clinically relevant Gram-positive organisms by use of the Bruker Biotyper Matrix-Assisted Laser Desorption Ionization-Time of Flight mass spectrometry system. J. Clin. Microbiol. 51:1421-1427. https://doi .org/10.1128/JCM.02680-12. 
Sampimon, O. C., T. J. G. M. Lam, D. J. Mevius, Y. H. Schukken, and R. N. Zadoks. 2011. Antimicrobial susceptibility of coagulase-negative staphylococci isolated from bovine milk samples. Vet. Microbiol. 150:173-179. https://doi.org/10.1016/j.vetmic.2011.01.017.

Seng, P., M. Drancourt, F. Gouriet, B. La Scola, P. Fournier, J. M. Rolain, and D. Raoult. 2009. Ongoing revolution in bacteriology: Routine identification of bacteria by matrix-assisted laser desorption ionization time-of-flight mass spectrometry. Clin. Infect. Dis. 49:543-551. https://doi.org/10.1086/600885.

Supré, K., F. Haesebrouck, R. N. Zadoks, M. Vaneechoutte, S. Piepers, and S. De Vliegher. 2011. Some coagulase-negative Staphylococcus species affect udder health more than others. J. Dairy Sci. 94:2329-2340. https://doi.org/10.3168/jds.2010-3741.

Tang, Y.-W., M. Sussman, D. Liu, I. Poxton, and J. Schwartzman. 2014. Molecular Medical Microbiology. Academic Press, Cambridge, MA.
Taponen, S., K. Supre, V. Piessens, E. Van Coillie, S. De Vliegher, and J. M. K. Koort. 2012. Staphylococcus agnetis sp. nov., a coagulasevariable species from bovine subclinical and mild clinical mastitis. Int. J. Syst. Evol. Microbiol. 62:61-65. https://doi.org/10.1099/ ijs.0.028365-0.

Vanderhaeghen, W., S. Piepers, F. Leroy, E. Van Coillie, F. Haesebrouck, and S. De Vliegher. 2014. Invited review: Effect, persistence, and virulence of coagulase-negative Staphylococcus species associated with ruminant udder health. J. Dairy Sci. 97:5275-5293. https://doi.org/10.3168/jds.2013-7775.

Zadoks, R., and J. Watts. 2009. Species identification of coagulasenegative staphylococci: Genotyping is superior to phenotyping. Vet. Microbiol. 134:20-28. https://doi.org/10.1016/j.vetmic.2008 .09.012. 